\title{
Strong Coupling of Antiferromagnetic Resonance with Subterahertz Cavity Fields
}

\author{
M. Białek $\odot,{ }^{1, *}, \dot{J}$ J. Zhang $\odot,{ }^{2, \ddagger} \mathrm{H} . \mathrm{Yu} \odot,^{2, \dagger}$ and J.-Ph. Ansermet ${ }^{1}$ \\ ${ }^{1}$ Institute of Physics, École Polytechnique Fédérale de Lausanne (EPFL), Lausanne 1015, Switzerland \\ ${ }^{2}$ Fert Beijing Institute, School of Microelectronics, Beijing Advanced Innovation Center for Big Data and Brain \\ Computing, Beihang University, Beijing 100191, China
}

(Received 4 January 2021; revised 9 March 2021; accepted 12 March 2021; published 9 April 2021)

\begin{abstract}
Strong coupling of electromagnetic cavity fields with antiferromagnetic spin waves in hematite $(\alpha$ $\mathrm{Fe}_{2} \mathrm{O}_{3}$ ) is achieved above room temperature. A cube of hematite is placed in a metallic tube and the transmission is measured, using a continuous-wave terahertz spectrometer. The spectra, collected as a function of the temperature, reveal the formation of magnetic polaritons.
\end{abstract}

DOI: 10.1103/PhysRevApplied.15.044018

\section{INTRODUCTION}

The hybrid nature of strongly coupled light-matter states, where the dissipation rate is lower than the exchange rate (Rabi frequency) [1], has attracted a lot of attention since the late 1980s [2]. In the decade of the 2000s, strong light-matter coupling has been investigated in the solid state [3], where the coupling of light interacting with $N$ resonators increases by a factor of $\sqrt{N}$ [4]. This regime might lead to device elements that may play a role in quantum devices [1,5-9]. In the 2010s, interest has turned toward magnon-photon coupling in ferromagnetic materials [10-22], as spins benefit from a relatively low coupling to their environment $[6,23]$. The achievement of strong coupling with antiferromagnets has the advantage of operating at frequencies in the terahertz $(\mathrm{THz})$ range [24,25], which allows access to a lot of interesting physics that is unreachable in ferromagnets [26-28]. A large variety of antiferromagnets are available and many of them show magnetic ordering above room temperature. The absence of stray fields could, in principle, allow for extremely dense packing of elements $[24,29]$. However, there are only reports on a few examples of weak magnon-photon coupling in antiferromagnets $[30,31]$. Strong coupling has only been achieved at low frequencies [32], in bulk samples [33,34], or via an indirect

*marcin.bialek@epfl.ch

†haiming.yu@buaa.edu.ch

$\$$ These authors contributed equally to this work.

Published by the American Physical Society under the terms of the Creative Commons Attribution 4.0 International license. Further distribution of this work must maintain attribution to the author(s) and the published article's title, journal citation, and DOI. coupling [35,36], while direct strong cavity-magnon coupling has not been shown so far [37]. One of the technical difficulties is to construct high-frequency cavities that are well coupled to waveguides. Here, we present a relatively simple implementation that allows strong coupling of the antiferromagnetic resonance and cavity electromagnetic modes.

In this paper, we show strong coupling between the electromagnetic fields of a three-dimensional cavity and spin waves in hematite $\left(\alpha-\mathrm{Fe}_{2} \mathrm{O}_{3}\right)$, a very common roomtemperature antiferromagnet. Hematite crystallizes in an approximately hexagonal structure with space group $R \overline{3} c$. Precise measurements show that the actual symmetry is monoclinic $\mathrm{C} 2 / c$ or $\mathrm{C}^{\prime} / c^{\prime}$ [38]. Below the Néel temperature $T_{N} \approx 955 \mathrm{~K}$ [39], the $\mathrm{Fe}^{3+}$ magnetic moments orient in an antiferromagnetic order. Above the spin-reorientation transition (the Morin phase transition) at about $T_{M} \approx 260$ $\mathrm{K}$ [40], the superexchange Dzialoshinskii-Moriya interaction leads to a canting of the two sublattices that gives rise to a net magnetization $\mathbf{m}$ [41], i.e., making this material a weak ferromagnet. Owing to the spin canting, the antiferromagnetic resonance has two modes, one of which is at a high frequency. This quasiantiferromagnetic resonance (qAFMR) mode is excited by dynamical magnetic field $\mathbf{h}$ parallel to the magnetization (h\| $\mathbf{m})$. At temperatures above room temperature, the width of the qAFMR is only about $1 \mathrm{GHz}$ and its frequency rises sharply with the temperature [40].

\section{EXPERIMENTAL}

Due to the development of frequency extenders for vector-network analyzers (VNAs), continuous-wave spectroscopic measurements up to $1.5 \mathrm{THz}$ can be rapidly conducted with a high frequency resolution and with a very high dynamic range [31,42-45]. 


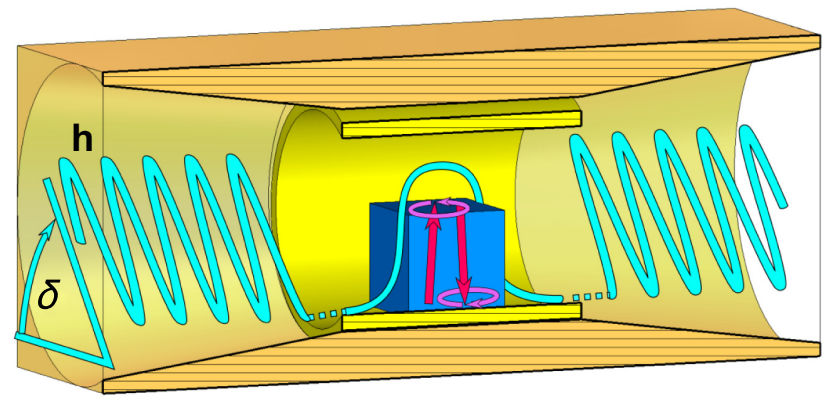

FIG. 1. A plane electromagnetic wave (blue wiggles) is focused into a cylinder cavity containing a hematite cube. The cavity magnetic field (blue curve) interacts with the resonance (violet circles) of two antiferromagnetic sublattices (red arrows). The transmitted linear polarization is measured parallel to that of the incident beam.

Out of a bulk natural single crystal, we cut a cube of $l_{s}=0.2 \mathrm{~mm}$ edge length, with faces in the $a, b$, and $c$ crystalline directions. The cube is placed inside a tube of length $l_{c}=2 \mathrm{~mm}$ and internal diameter $2 r_{c}=0.58 \mathrm{~mm}$, cut out of a gold-plated stainless steel needle. The needle is wrapped with Teflon tape to prevent the cube from dropping out of the tube. The tube with the cube in it is inserted into a copper holder composed of two cones (cone angle, about $60^{\circ}$ ), which focuses the terahertz beam into the cavity (Fig. 1). The terahertz beam propagates between the source, sample holder, and detector in oversized metallic waveguides, 8 $\mathrm{mm}$ in diameter, matching the larger diameter of the cones. The holder is placed between Peltier elements that control its temperature, which is monitored with a K-type thermocouple inserted in a hole on its side. The detector measures the transmitted power and phase of the terahertz electric field. Temperature scans start from the highest temperature, with a step of $\Delta T=-0.25 \mathrm{~K}$. This temperature step is chosen so that the frequency change is smaller than the line width of the resonance. After stabilizing the sample temperature $T$, we measure the transmission as a function of the radiation frequency $f$.

The source emits a linearly polarized beam of terahertz radiation and the detector detects only radiation of linear polarization that matches its waveguide, i.e., it acts like a polarization filter. Rotation of the polarization plane is possible by rotating the source and detector about the optical axis. We define the polarization angle $\delta$ as the angle of the radiation $h$ field with respect to the plane of the optical table (Fig. 1). We measure the transmission in a polarization-angle range of $\delta=-90^{\circ}$ to $+90^{\circ}$.

\section{RESULTS AND ANALYSIS}

In Fig. 2(a), we can see an avoided crossing of the cavity mode at $f_{1} \approx 242.5 \mathrm{GHz}$ with the qAFMR mode, the frequency of which $f_{r}(T)$ rises approximately linearly with the temperature (see the Appendix A). This result is

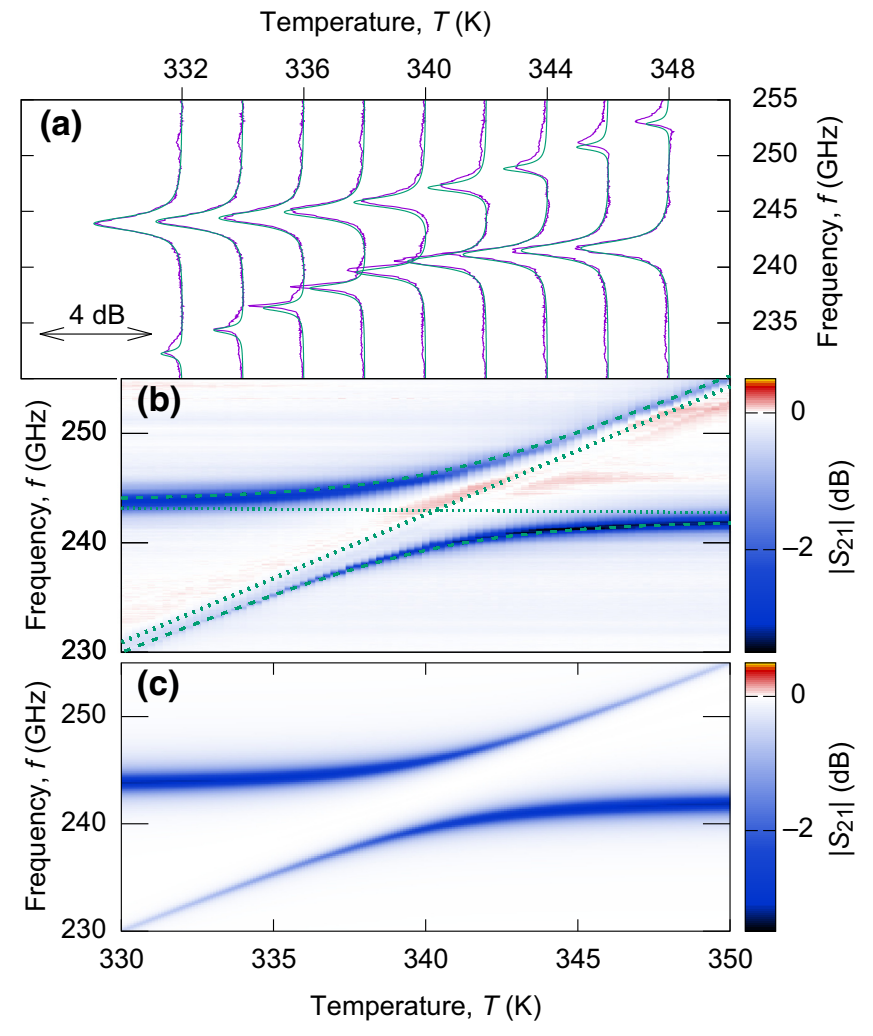

FIG. 2. The normalized transmission magnitude at polarization angle $\delta=30^{\circ}$. (a) The measured spectra (violet lines) and fit using Eq. (1) (green lines). (b) A map of the spectra: the green dashed lines show interacting and noninteracting modes fitted using Eq. (3). (c) A map of the spectra fitted using Eq. (1).

obtained at polarization angle $\delta=30^{\circ}$. We fit the observed spectra using the equation developed in the framework of input-output theory $[10,46]$ :

$$
S_{21}=1+\frac{a_{1}}{i\left[f-f_{1}(T)\right]-\frac{\kappa_{1}}{2}+\frac{G_{1}^{2}}{i\left[f-f_{r}(T)\right]-g / 2}},
$$

where $a_{1}=(0.32-i 0.02) \mathrm{GHz}$ is a complex parameter describing the coupling of the cavity with the source and the detector (see the Appendix C). The parameter $f_{1}(T) \approx$ $242.5 \mathrm{GHz}$ is the observed frequency of the first cavity mode and $\kappa_{1}=2.1 \mathrm{GHz}$ describes its width, $2 G_{1}=6.2$ $\mathrm{GHz}$ is the minimum splitting between the upper and lower polariton branches, and $g=0.5 \mathrm{GHz}$ describes the qAFMR width. With these parameters, we can determine the cooperativity factor

$$
C=\frac{4 G_{1}^{2}}{\kappa_{1} g} \approx 40
$$

that is, the square of a ratio of the Rabi splitting $\left(2 G_{1}\right)$ and the losses of the polariton states. This means that the splitting is about 6 times larger than the line width. According to the harmonic coupling model, the upper and lower 
polariton frequencies are given by $[12,47]$,

$$
f_{ \pm}=\frac{1}{2}\left(f_{1}+f_{r} \pm \sqrt{\left(f_{1}-f_{r}\right)^{2}+4 g_{i}^{2} f_{1}}\right) \text {. }
$$

The coupled mode frequencies $f_{ \pm}$are drawn on Fig. 2(b) as green dotted lines, with $2 g_{i} \sqrt{f_{1}}=2 G_{1}=6.2 \mathrm{GHz}$, as well as the noninteracting mode frequencies $f_{r}(T)$ and $f_{1}(T)$. Clearly, this prediction matches that of Eq. (1) [Fig. 2(b)]. The discrepancies between fits using Eq. (1) and the data [Fig. 2(a)] may arise from interactions with other cavity modes, as we discuss in further sections of this paper.

\section{A. Microscopic model}

The coupling strength can be calculated using a microscopic model [12]:

$$
g_{i}=\frac{g_{s} \mu_{B}}{2 h} \sqrt{\frac{\mu_{0} h}{2} \rho \frac{V_{s}}{V_{c}}},
$$

where $g_{s}=2, \rho$ is the density of resonators in a hematite cube of volume $V_{s}=l_{s}^{3}$ and $V_{c}$ is the volume of the metal tube, $V_{c}=\pi r_{c}^{2} l_{c} \approx 0.528 \mathrm{~mm}^{3}$. In Eq. (4), each magnon is coupled to the electromagnetic cavity mode with a coupling strength $g_{s} \mu_{B} B_{0} / 2 h$ [48], where $B_{0}=$ $\sqrt{\mu_{0} h f_{1} / 2 V_{c}} \approx 4.4 \times 10^{-10} \mathrm{~T}$ is the magnetic component of the vacuum fluctuations [49] (here, $f_{1}=242.5 \mathrm{GHz}$ ). This is a few orders of magnitude smaller than the amplitude of the terahertz field in our experiment. The collective coupling strength of $N=\rho V_{s}$ oscillators is increased by a factor $\sqrt{N}[4,12,14,35,48]$; thus $g_{i}$ depends only on the oscillator density $\rho$ and the ratio of the crystal volume to the cavity volume [12,35], that is $V_{s} / V_{c} \approx 1.51 \times 10^{-2}$.

We assume that the density of oscillators in hematite is $\rho=5 \rho_{\mathrm{Fe}}[50,51]$, where $\rho_{\mathrm{Fe}}=3.987 \times 10^{28} \mathrm{~m}^{-3}$ [52] and the factor of 5 comes from the magnetic moment of $\mathrm{Fe}^{3+}$ ions [53]. The density of iron atoms in hematite is quite high compared to that of many common antiferromagnets. Hence, it is a good material with which to achieve strong coupling. Taking this into account gives $g_{i}=1.57 \times 10^{4} \mathrm{~Hz}^{1 / 2}$, i.e., the splitting is predicted to be $2 G_{p}=2 g_{i} \sqrt{f_{1}} \approx 15.4 \mathrm{GHz}$ for the cavity mode at $f_{1}=242.5 \mathrm{GHz}$. The observed splitting $2 G_{1}=6.2 \mathrm{GHz}$ is about $40 \%$ of that value. This discrepancy results from an imperfect matching of the electromagnetic mode with the antiferromagnetic resonance in the hematite cube, which is excited by fields parallel to the weak ferromagnetic moment. As confirmed in the simulation discussed below (Fig. 5), the cavity magnetic field in the volume of the cube does not excite all the spins with full amplitude. We calculate that in the first mode, the magnetic field component $h_{z}^{2}$ makes about $78 \%$ of all the integrated magnetic field energy in the cube volume (Table I). The remaining difference between the predicted and the observed splitting must result from the magnetic moment of the cube being titled away from the $z$ axis.

\section{B. Angular dependence}

We find that the parameter $a_{1}$ strongly depends on the polarization angle $\delta$ [Fig. 3(a)]. This parameter describes the coupling between the cavity and the rest of the spectrometer, i.e., constructive or destructive interference between the cavity and the experimental setup. The result presented in Fig. 1(a), obtained at $\delta=30^{\circ}$, shows a minimum in transmission that is accounted for with $a_{1}$ having a small imaginary part and a positive real part. The dependence of $a_{1}$ on the polarization angle [Fig. 3(a)] is not an intrinsic property of the cavity but depends on the coupling of the cavity with the experimental setup. As a consequence, after it is rearranged, we obtain a slightly different value for $a_{1}$ [Fig. 1(b)]. Thus, the result obtained at $\delta=$ $-75^{\circ}$ [Fig. 3(c)] under conditions (a) shows a dispersive line shape that is accounted for by a negative imaginary $a_{1}$ [Fig. 3(d)]. In contrast, the result obtained at $\delta=-60^{\circ}$ [Fig. 3(e)] under conditions (b) shows constructive interference that is explained by an almost entirely real negative $a_{1}$ [Fig. 3(f)]. Under the same conditions, at $\delta=70^{\circ}$ a weak inverted dispersive line shape is observed [Fig. 3(g)], which is accounted for with a small positive imaginary $a_{1}$ [Fig. 3(h)].

\section{Higher cavity modes}

The raw data for the spectra are dominated by multiple instances of interference that are not strongly interacting with the cavity and are weakly temperature dependent. We normalize both the measured spectra and the fitted functions to a base frequency that is temperature dependent and that passes though the middle of the interaction.

TABLE I. The predicted mode frequencies, selection rules, and mean magnetic field in the cube.

\begin{tabular}{cccccccccc}
\hline \hline & \multicolumn{3}{c}{ Cavity } & \multicolumn{5}{c}{ Mean $h$ field in the cube (arbitrary units) } \\
$j$ & $f_{j}(\mathrm{GHz})$ & $e$ field & $h_{x}$ & $h_{y}$ & $h_{z}$ & $\left|h_{x}\right|$ & $\left|h_{y}\right|$ & $\left|h_{z}\right|$ & $|\mathbf{h}|$ \\
\hline 1 & 247 & $x$ & 0.01 & -0.01 & 2.29 & 0.42 & 0.66 & $\mathbf{2 . 3 9}$ \\
2 & 258 & $y$ & 0.12 & 0.00 & 0.00 & 0.96 & 0.09 & 1.30 & 1.76 \\
3 & 272 & $z$ & 2.51 & 0.00 & -0.01 & $\mathbf{2 . 6 1}$ & 0.75 & 0.63 & 3.05 \\
4 & 297 & - & 0.00 & 2.30 & 0.01 & 0.72 & $\mathbf{2 . 5 1}$ & 0.90 \\
5 & 329 & $x$ & 0.00 & 0.00 & -1.00 & 0.54 & 0.43 & $\mathbf{1 . 8 7}$ & 2.15 \\
\hline \hline
\end{tabular}


This reduces artifacts at the cavity mode frequency that would show if, instead, we normalized to, for example, the first recorded spectrum. We could eliminate this interference background even more effectively by calculating temperature-derivative spectra, i.e., by subtracting successive spectra from one another, thus having amplitude derivatives,

$$
\frac{d\left|S_{21}\right|}{d T}[d B]=\frac{20}{\Delta T} \log _{10} \frac{\left|S_{21}(f, T+\Delta T)\right|}{\left|S_{21}(f, T)\right|},
$$

and phase derivatives,

$$
\frac{d\left(\arg S_{21}\right)}{d T}=\frac{\arg S_{21}(f, T+\Delta T)-\arg S_{21}(f, T)}{\Delta T} .
$$

The advantage of looking at temperature-differential spectra is that it is possible to see small features, such as a weak middle line passing through the interaction region [Figs. 4(a) and 4(c)] that is almost invisible in normalized spectra. This middle line can be qualitatively explained by taking into account the second cavity mode at $f_{2}=258.4$ $\mathrm{GHz}$. We can take this into account by assuming that both modes interact independently with the magnetic resonance mode:

$$
S_{21}=1+\sum_{j=1}^{2} \frac{a_{j}}{i\left[f-f_{j}(T)\right]-\frac{\kappa_{j}}{2}+\frac{G_{j}^{2}}{i\left[f-f_{r}(T)\right]-g / 2}},
$$

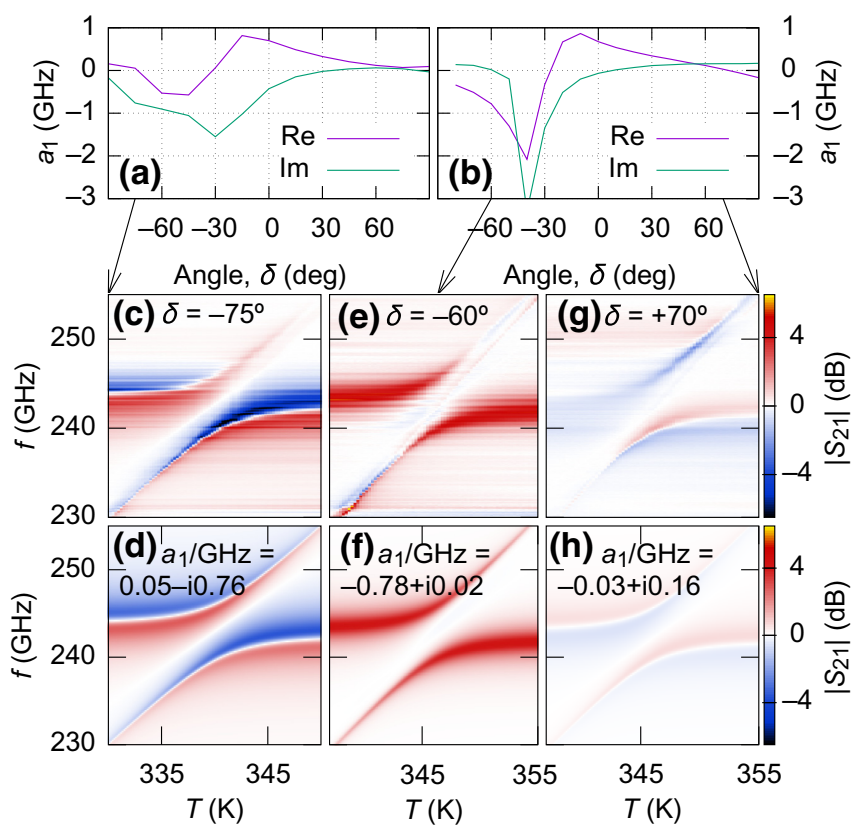

FIG. 3. (a),(b) The angular dependence of $a_{1}$ [Eq. (1)] in two configurations of the experiment. (c) The normalized $\left|S_{21}\right|$ at $\delta=$ $-75^{\circ}$ in configuration (a). (e) The normalized $\left|S_{21}\right|$ at $\delta=-60^{\circ}$ and (g) $\delta=70^{\circ}$, both in configuration (b). (d),(f),(h) Fits using Eq. (1).

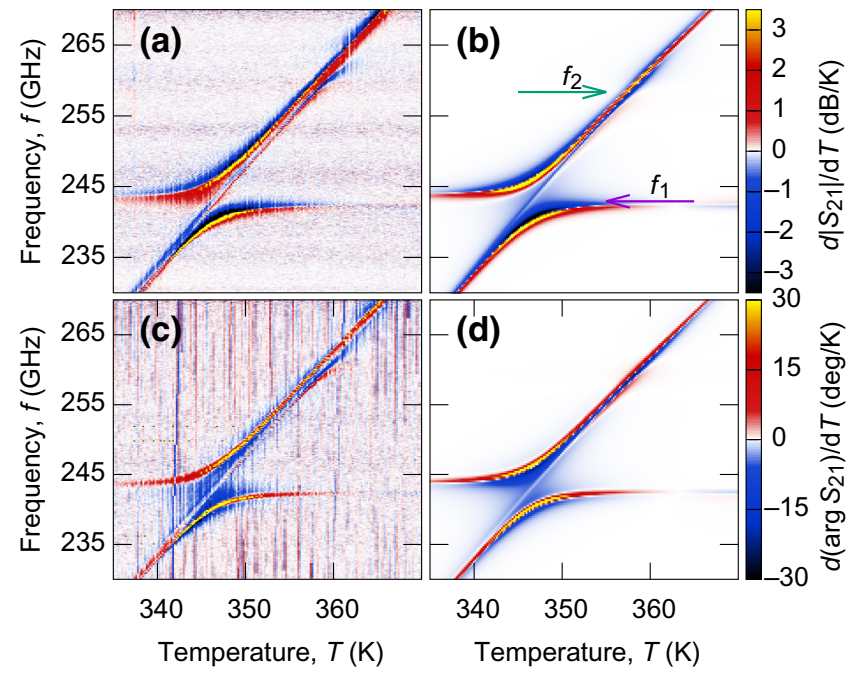

FIG. 4. The temperature-derivative spectra: (a) magnitude and (c) phase. (b),(d) Corresponding fits using Eq. (7).

In Fig. 4(b), we present the temperature-differential of Eq. (7), where we set $a_{2}=(4.5-i 1.1) \mathrm{GHz}, G_{2}=0.56 \mathrm{GHz}$, and $\kappa_{2}=14.7 \mathrm{GHz}$. These values are consistent with our idea of a weak interaction with the second cavity mode, which has a lower quality factor than the first mode. This simple model is not fully sufficient above $250 \mathrm{GHz}$. We think that a more precise estimate might require taking into account other weakly interacting cavity modes or interactions between cavity modes that are mediated by the magnetic resonance. We find that the observed phase temperature-differential spectra [Fig. 4(c)] are reproduced by the same set of parameters [Fig. 4(d)], using Eqs. (7) and (6).

\section{Electrodynamic simulations of cavity modes}

We identify the cavity modes using numerical electrodynamics field-simulation software (CST Microwave Studio). We model the cavity as a metallic cylinder containing an isotropic dielectric cube (Fig. 5). We assume that the dielectric constant of hematite is 19.1 at $350 \mathrm{~K}$, as determined from our measurements on bulk samples [54]. We calculate the dependence of the modes on the size and position of the cube inside the cylinder (see the Appendix B). The actual size of the cube is the smallest we can achieve by our cutting process.

In Table I, we give the expected frequencies of the first five modes and the dominant directions of the electric field in the cavity, as well as the dominant direction of the magnetic field in the cube. Only the fifth mode has a similar symmetry to that of the first mode and is able to excite antiferromagnetic resonance in the sample. This suggests that the magnetization vector $\mathbf{m}$ in the cube is aligned along the $z$ axis of the cavity during the experiment. Under this 


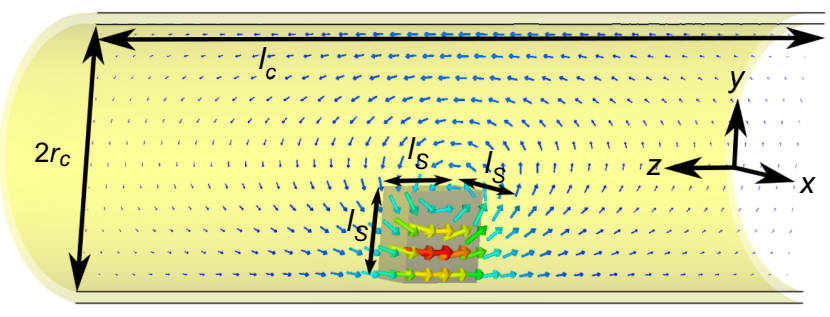

FIG. 5. A model of the cavity, drawn to scale, in cross section. The arrows show the strength and the direction of the magnetic field of the first cavity mode on the $x$ plane.

assumption, modes 2-4 do not excite the magnetic resonance and thus should only weakly hybridize with it. This agrees with our observation, in Fig. 6, that only the fifth mode produces some strong coupling, though not as clear as the coupling to the first mode. This may be due to a more complex distribution of the cavity magnetic field in the cube, i.e., the ratio of $h_{z} /\left|h_{z}\right| \approx 0.53$ is lower than in the case of the first mode (Table I). This means that some of the spins in the cube are excited out of phase.

\section{SUMMARY}

In conclusion, we observe strong coupling between the qAFMR of a hematite cube $\left(\alpha-\mathrm{Fe}_{2} \mathrm{O}_{3}\right)$ located in a cavity near room temperature. We show a method of achieving a strong light-matter coupling regime in antiferromagnets that is applicable to a broad range of these materials. The avoided crossing, which is characteristic of polariton dynamics, occurs near $243 \mathrm{GHz}$. The cooperativity $C$, which is a measure of the coupling strength with respect to both the magnetic resonance and the cavity line widths [Eq. (2)], is estimated at 40.

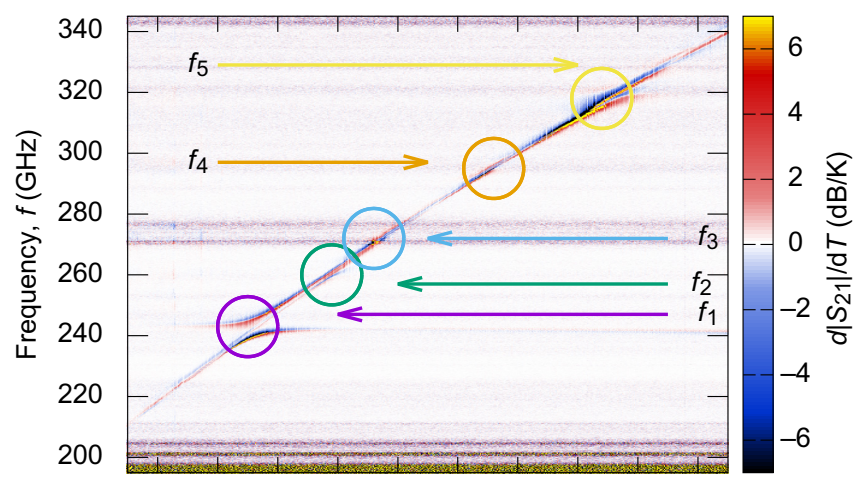

$330340350360370380390400 \quad 410420$

Temperature, $T(\mathrm{~K})$

FIG. 6. The temperature-derivative spectra over a broad temperature range. The arrows mark the expected frequencies of the cavity modes and the circles mark the observed interactions.

\section{ACKNOWLEDGMENTS}

We would like to thank Claude Amendola for help in fabricating samples. Support by the Sino-Swiss Science and Technology Cooperation (SSSTC) under Grant No. EG-CN_02_032019 is gratefully acknowledged. The VNA and frequency extenders were funded by the École polytechnique fédérale de Lausanne (EPFL) and the Swiss National Science Foundation (SNSF) Research Equipment (R'Equip) under Grant No. 206021_144983.

\section{APPENDIX A: TEMPERATURE DEPENDENCE OF THE ANTIFERROMAGNETIC RESONANCE AND THE FIRST CAVITY MODE}

Figure 7 shows the observed temperature dependence of the first mode, which we determine as

$$
f_{1}(T)=f_{1}^{345}+f_{1}^{T}\left(T-T_{1}\right)
$$

where $f_{1}^{345}=243 \mathrm{GHz}, T_{1}=345 \mathrm{~K}$, and $f_{1}{ }^{T}=-2.1 \times$ $10^{-2} \mathrm{GHz} / \mathrm{K}$. This dependence is caused by the dielectric constant of hematite increasing with rising temperature. For the cube, this dependence on the dielectric constant is predicted to be small [Fig. 8(f)].

In configuration (a) [Figs. 2(c) and 3(d)], the temperature dependence of the antiferromagnetic resonance is fitted with a linear function

$$
f_{r}(T)=f_{r}^{330}+f_{r}^{T}\left(T-T_{r}\right),
$$

where $f_{r}^{330}=230.7 \mathrm{GHz}, T_{r}=330 \mathrm{~K}$, and $f_{r}^{T}=1.176$ $\mathrm{GHz} / \mathrm{K}$. In configuration (b) [Fig. 3(fh)], we fit $f_{r}^{330}=$ $220.1 \mathrm{GHz}$ and $f_{r}^{T}=1.410 \mathrm{GHz} / \mathrm{K}$. In Figs. 4(b) and 4(d), where we show the temperature dependence on a broader scale, it is necessary to assume that the frequency of the qAFMR is described by a parabolic function

$$
f_{r}(T)=f_{r}^{330}+f_{r}^{T}\left(T-T_{r}\right)+f_{r}^{T 2}\left(T-T_{r}\right)^{2},
$$

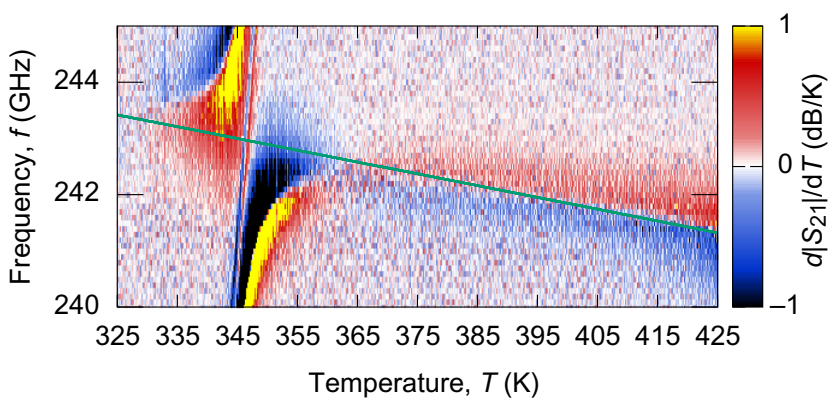

FIG. 7. A close-up of the first cavity mode in an oversaturated scale. The green line shows the assumed linear temperature dependence of the first mode frequency. 

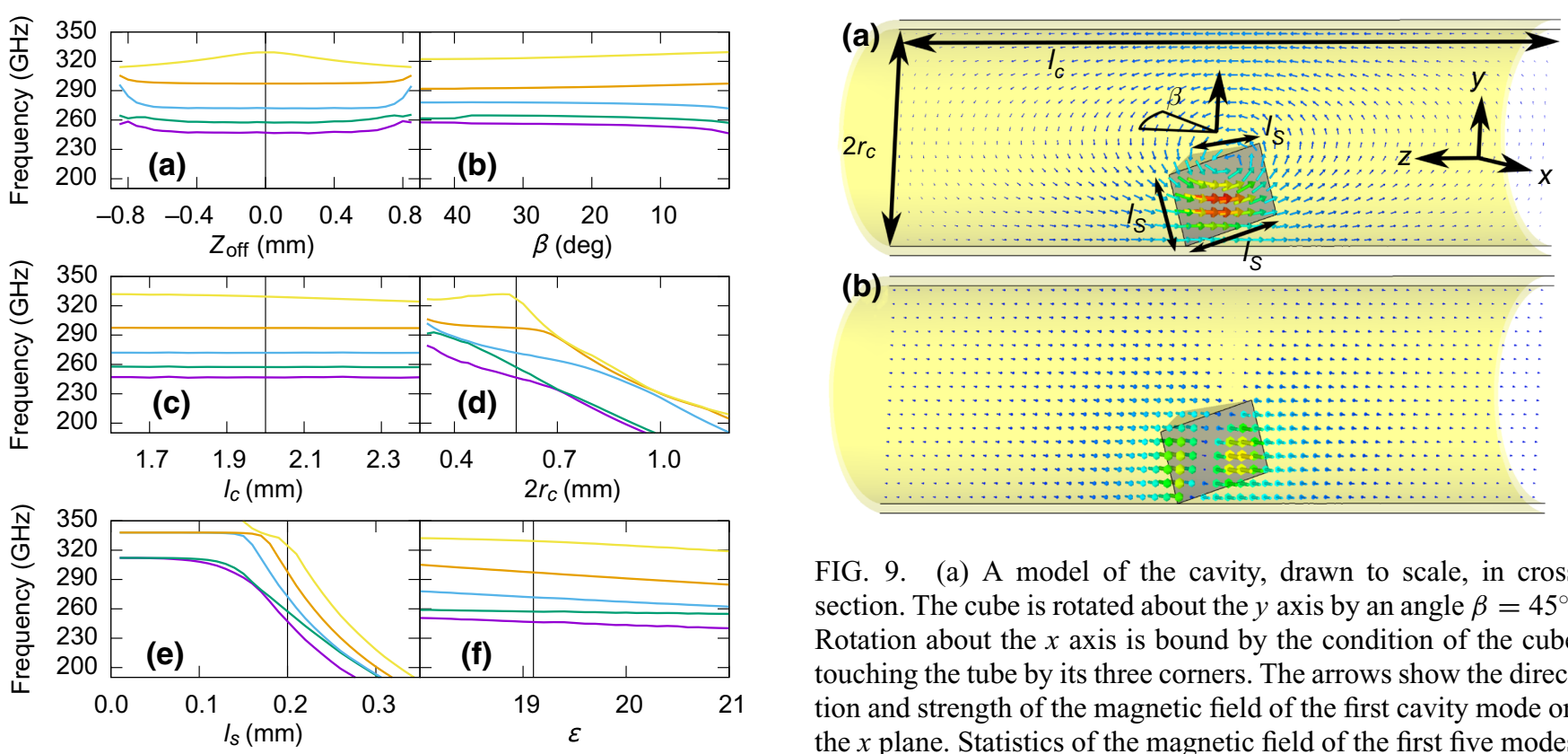

FIG. 9. (a) A model of the cavity, drawn to scale, in cross section. The cube is rotated about the $y$ axis by an angle $\beta=45^{\circ}$. Rotation about the $x$ axis is bound by the condition of the cube touching the tube by its three corners. The arrows show the direction and strength of the magnetic field of the first cavity mode on the $x$ plane. Statistics of the magnetic field of the first five modes are presented in Table II. (b) The strength and the direction of the magnetic field in the second mode the cavity on the $x$ plane.

FIG. 8. The dependence of the mode frequencies on (a) the displacement of the cube from the center of the tube, (b) the rotation of the cube about the $y$ axis, (c) the length of the tube, (d) the diameter of the tube, (e) the cube edge length, and (f) the dielectric constant of the cube. In (a), (c), (d), (e), and (f), the vertical lines mark nominal values of these parameters.

where $f_{r}^{330}=218.4 \mathrm{GHz}, \quad T_{r}=330 \mathrm{~K}, f_{r}^{T}=1.596$ $\mathrm{GHz} / \mathrm{K}$, and $f_{r}^{T 2}=-5.15 \times 10^{-3} \mathrm{GHz} / \mathrm{K}^{2}$. These differences of $f_{r}(T)$ result from different placements of the thermocouple in the holder and, thus, a different temperature calibration.

\section{APPENDIX B: FREQUENCY DEPENDENCE OF MODES ON CAVITY GEOMETRY}

The size of the cube $l_{s}$ is limited by our cutting process. At this smallest size, the mode frequencies are governed mostly by the size of the cube $l_{s}[$ Fig. $8(\mathrm{e})]$ and, to a lesser extent, by the diameter of the tube $2 r_{c}$ [Fig. 8(d)]. The length of the tube $l_{c}$ is irrelevant in the range of about 1.5-2.5 mm [Fig. 8(c)]. For a given cube size, we choose the diameter of the tube such that the first and second modes are well split [Fig. 8(d)]. We find that a random position of the cube in the needle spans a mode frequency range of at most $15 \mathrm{GHz}$ [Figs. 8(a) and 8(b)]. In Fig. 9 and Table II, we show that the magnetic field of the cavity modes has similar symmetry when the cube is rotated by $\beta=45$. In other calculations [Figs. $8(\mathrm{c})-8(\mathrm{f})$ ], the cube is assumed to be in the center of the needle $\left(Z_{\text {off }}=0\right)$ and the edges parallel to its axis $(\beta=0)$. The calculated frequency of the first mode is a few gigahertz higher than the observed one. This might be due to inaccuracy in the cube dimensions. We observe the biggest discrepancy with

the predicted and observed frequencies of the fifth mode (Fig. 6). This could be related to the fact that it is the mode that shows the strongest dependence on displacement of the cube from the cavity center [Fig. 8(a)].

\section{APPENDIX C: TWO-PORT ANALYSIS}

Let us represent a cavity as a device with two ports. At each port, we distinguish incoming and outgoing waves. The total voltage and current at each port are given by

$$
V_{i}=V_{i}^{+}+V_{i}^{-}, \quad I_{i}=I_{i}^{+}+I_{i}^{-} \quad(i=1,2)
$$

One way of characterizing the cavity is to specify the parameters $A, B, C$, and $D$ defined by

$$
\left(\begin{array}{l}
V_{1} \\
I_{1}
\end{array}\right)=\left(\begin{array}{ll}
A & B \\
C & D
\end{array}\right)\left(\begin{array}{l}
V_{2} \\
I_{2}
\end{array}\right)
$$

For a two-port device in which each port sees the common impedance $Z_{3}$, the parameters $A, B, C$, and $D$ parameters are given by [55]

$$
A=1, \quad B=0, \quad C=\frac{1}{Z_{3}}, \quad D=1 .
$$

The scattering matrix is defined by

$$
\left(\begin{array}{l}
V_{1}^{-} \\
V_{2}^{-}
\end{array}\right)=\left(\begin{array}{ll}
S_{11} & S_{12} \\
S_{21} & S_{22}
\end{array}\right)\left(\begin{array}{l}
V_{1}^{+} \\
V_{2}^{+}
\end{array}\right)
$$


TABLE II. The predicted mode frequencies for the cube rotated by $\beta=45^{\circ}$ [Fig. 9(a)]. The selection rules and the mean magnetic field in the cube are very close to that of $\beta=0^{\circ}$ (Table I).

\begin{tabular}{cccccccccc}
\hline \hline & & \multicolumn{3}{c}{ Cavity } & \multicolumn{5}{c}{ Mean $h$ field in the cube (arbitrary units) } \\
$j$ & $f_{j}(\mathrm{GHz})$ & $e$ field & $h_{x}$ & $h_{y}$ & $h_{z}$ & $\left|h_{x}\right|$ & $\left|h_{y}\right|$ & $\left|h_{z}\right|$ & $|\mathbf{h}|$ \\
\hline 1 & 258 & $x$ & 0.00 & -0.02 & 2.20 & 0.43 & 0.62 & $\mathbf{2 . 3 2}$ \\
2 & 262 & $y$ & 0.33 & 0.00 & 0.01 & 0.93 & 0.31 & 1.24 & 1.74 \\
3 & 278 & $z$ & -2.55 & 0.00 & 0.00 & $\mathbf{2 . 6 8}$ & 0.63 & 0.67 & 3.06 \\
4 & 292 & - & 0.00 & 2.45 & 0.00 & 0.68 & $\mathbf{2 . 6 4}$ & 0.77 & 3.15 \\
5 & 322 & $x$ & 0.00 & 0.00 & 1.29 & 0.58 & 0.69 & $\mathbf{2 . 2 8}$ & 2.71 \\
\hline \hline
\end{tabular}

In particular, the $S_{21}$ parameter is given by

$$
S_{21}=\left.\frac{V_{2}^{-}}{V_{1}^{+}}\right|_{V_{2}^{+}=0} .
$$

It is straightforward to calculate that

$$
S_{21}=\frac{1}{1+Z_{0} /\left(2 Z_{3}\right)}
$$

where $Z_{0}$ is the transmission line (or waveguide) characteristic impedance at the ports.

Let us apply this formalism to analyze a magnetic material located inside the cavity. We represent the cavity by discrete $R, L$, and $C$ components in series. We assume that the measurement setup is such that away from a resonance of the cavity or from a resonance of the material in it, the signal propagates through the cavity without loss, i.e., $S_{21}=1$. We have

$$
Z_{3}=i \omega L+\frac{1}{i \omega C}+R
$$

We assume that the $R L C$ circuit is designed so that the transmission is nearly one at all frequencies, i.e., $Z_{3} \gg Z_{0}$. Then, Eq. (C6) yields

$$
S_{21} \approx 1-\frac{Z_{0}}{2 Z_{3}}
$$

We take the presence of the material in the cavity into account by assuming that the inductance $L$ is filled entirely with a medium of an effective permittivity $\mu$, written in the form of a Lorentzian line shape, so as to account for the magnetic resonance in the material

$$
L=\mu L_{0}=\left(1+\frac{\Delta \mu \omega_{m}^{2}}{\omega_{m}^{2}-\omega^{2}-i \omega \gamma_{m}}\right) L_{0}
$$

where $L_{0}$ is the inductance of an empty cavity, $\Delta \mu$ is a unitless parameter that characterizes the coupling of the material to the cavity, $\omega_{m}$ is its frequency, and $\gamma_{m}$ defines its width. Using $Z_{3}$ given by Eqs. (C7) and (C9), Eq. (C8) gives

$$
S_{21}=1-\frac{i \omega Z_{0} C / 2}{1+i \omega R C-\omega^{2} C L_{0}\left(1+\frac{\Delta \mu \omega_{m}^{2}}{\omega_{m}^{2}-\omega^{2}-i \omega \gamma_{m}}\right)} .
$$

Let us call $\omega_{c}^{2}=1 /\left(L_{0} C\right)$ in reference to the resonance of the empty cavity. We consider a situation in which $\omega, \omega_{c}$, and $\omega_{m}$ are close, so we can approximate

$$
\omega^{2}-\omega_{c}^{2}=\left(\omega-\omega_{c}\right)\left(\omega+\omega_{c}\right) \approx 2 \omega_{c}\left(\omega-\omega_{c}\right) .
$$

Since the resonances are sharp, $\omega$ can be replaced by $\omega_{c}$ when $\omega$ is not in a term that leads to a divergence (in the absence of damping). Thus, we can write, as in Ref. [10],

$$
S_{21}=1+\frac{a}{i\left(\omega-\omega_{c}\right)-\frac{\kappa}{2}+\frac{G^{2}}{i\left(\omega-\omega_{m}\right)-\gamma_{m} / 2}},
$$

where $a=\omega_{c}^{2} Z_{0} C / 4, G^{2}=\omega_{c} \omega_{m} \Delta \mu / 4$ and $\kappa=\omega_{c}^{2} R C$.

[1] P. Törmä and W. L. Barnes, Strong coupling between surface plasmon polaritons and emitters: A review, Rep. Prog. Phys. 78, 013901 (2014).

[2] G. Rempe, H. Walther, and N. Klein, Observation of Quantum Collapse and Revival in a One-Atom Maser, Phys. Rev. Lett. 58, 353 (1987).

[3] G. Khitrova, H. M. Gibbs, M. Kira, S. W. Koch, and A. Scherer, Vacuum Rabi splitting in semiconductors, Nat. Phys. 2, 81 (2006).

[4] M. G. Raizen, R. J. Thompson, R. J. Brecha, H. J. Kimble, and H. J. Carmichael, Normal-Mode Splitting and Linewidth Averaging for Two-State Atoms in an Optical Cavity, Phys. Rev. Lett. 63, 240 (1989).

[5] J. Kasprzak, M. Richard, S. Kundermann, A. Baas, P. Jeambrun, J. M. J. Keeling, F. M. Marchetti, M. H. Szymańska, R. André, J. L. Staehli, V. Savona, P. B. Littlewood, B. Deveaud, and L. S. Dang, Bose-Einstein condensation of exciton polaritons, Nature 443, 409 (2006).

[6] D. D. Awschalom and M. E. Flatté, Challenges for semiconductor spintronics, Nat. Phys. 3, 153 (2007). 
[7] D. S. Dovzhenko, S. V. Ryabchuk, Y. P. Rakovich, and I. R. Nabiev, Light-matter interaction in the strong coupling regime: Configurations, conditions, and applications, Nanoscale 10, 3589 (2018).

[8] A. F. Kockum, A. Miranowicz, S. De Liberato, S. Savasta, and F. Nori, Ultrastrong coupling between light and matter, Nat. Rev. Phys. 1, 19 (2019).

[9] K. Roux, H. Konishi, V. Helson, and J.-P. Brantut, Strongly correlated fermions strongly coupled to light, Nat. Commun. 11, 2974 (2020).

[10] D. I. Schuster, A. P. Sears, E. Ginossar, L. DiCarlo, L. Frunzio, J. J. L. Morton, H. Wu, G. A. D. Briggs, B. B. Buckley, D. D. Awschalom, and R. J. Schoelkopf, High-Cooperativity Coupling of Electron-Spin Ensembles to Superconducting Cavities, Phys. Rev. Lett. 105, 140501 (2010).

[11] E. Abe, H. Wu, A. Ardavan, and J. J. L. Morton, Electron spin ensemble strongly coupled to a three-dimensional microwave cavity, Appl. Phys. Lett. 98, 251108 (2011).

[12] H. Huebl, C. W. Zollitsch, J. Lotze, F. Hocke, M. Greifenstein, A. Marx, R. Gross, and S. T. B. Goennenwein, High Cooperativity in Coupled Microwave Resonator Ferrimagnetic Insulator Hybrids, Phys. Rev. Lett. 111, 127003 (2013).

[13] X. Zhang, C.-L. Zou, L. Jiang, and H. X. Tang, Strongly Coupled Magnons and Cavity Microwave Photons, Phys. Rev. Lett. 113, 156401 (2014).

[14] Y. Tabuchi, S. Ishino, T. Ishikawa, R. Yamazaki, K. Usami, and Y. Nakamura, Hybridizing Ferromagnetic Magnons and Microwave Photons in the Quantum Limit, Phys. Rev. Lett. 113, 083603 (2014).

[15] Y. Tabuchi, S. Ishino, A. Noguchi, T. Ishikawa, R. Yamazaki, K. Usami, and Y. Nakamura, Coherent coupling between a ferromagnetic magnon and a superconducting qubit, Science 349, 405 (2015).

[16] X. Zhang, C.-L. Zou, N. Zhu, F. Marquardt, L. Jiang, and H. X. Tang, Magnon dark modes and gradient memory, Nat. Commun. 6, 8914 (2015).

[17] X. Zhang, N. Zhu, C.-L. Zou, and H. X. Tang, Optomagnonic Whispering Gallery Microresonators, Phys. Rev. Lett. 117, 123605 (2016).

[18] Y. Li, T. Polakovic, Y.-L. Wang, J. Xu, S. Lendinez, Z. Zhang, J. Ding, T. Khaire, H. Saglam, R. Divan, J. Pearson, W.-K. Kwok, Z. Xiao, V. Novosad, A. Hoffmann, and W. Zhang, Strong Coupling between Magnons and Microwave Photons in On-Chip Ferromagnet-Superconductor ThinFilm Devices, Phys. Rev. Lett. 123, 107701 (2019).

[19] C. A. Potts and J. P. Davis, Strong magnon-photon coupling within a tunable cryogenic microwave cavity, Appl. Phys. Lett. 116, 263503 (2020).

[20] D. Lachance-Quirion, S. P. Wolski, Y. Tabuchi, S. Kono, K. Usami, and Y. Nakamura, Entanglement-based single-shot detection of a single magnon with a superconducting qubit, Science 367, 425 (2020).

[21] Y. Li, W. Zhang, V. Tyberkevych, W.-K. Kwok, A. Hoffmann, and V. Novosad, Hybrid magnonics: Physics, circuits, and applications for coherent information processing, J. Appl. Phys. 128, 130902 (2020).

[22] B. Bhoi, S.-H. Jang, B. Kim, and S.-K. Kim, Broadband photon-magnon coupling using arrays of photon resonators, J. Appl. Phys. 129, 083904 (2021).
[23] T. Niemczyk, F. Deppe, H. Huebl, E. P. Menzel, F. Hocke, M. J. Schwarz, J. J. Garcia-Ripoll, D. Zueco, T. Hümmer, E. Solano, A. Marx, and R. Gross, Circuit quantum electrodynamics in the ultrastrong-coupling regime, Nat. Phys. 6, 772 (2010).

[24] T. Jungwirth, J. Sinova, A. Manchon, X. Marti, J. Wunderlich, and C. Felser, The multiple directions of antiferromagnetic spintronics, Nat. Phys. 14, 200 (2018).

[25] J. Li, C. B. Wilson, R. Cheng, M. Lohmann, M. Kavand, W. Yuan, M. Aldosary, N. Agladze, P. Wei, M. S. Sherwin, and J. Shi, Spin current from sub-terahertz-generated antiferromagnetic magnons, Nature 578, 70 (2020).

[26] J. Li, H. T. Simensen, D. Reitz, Q. Sun, W. Yuan, C. Li, Y. Tserkovnyak, A. Brataas, and J. Shi, Observation of Magnon Polarons in a Uniaxial Antiferromagnetic Insulator, Phys. Rev. Lett. 125, 217201 (2020).

[27] D. Reitz, J. Li, W. Yuan, J. Shi, and Y. Tserkovnyak, Spin Seebeck effect near the antiferromagnetic spin-flop transition, Phys. Rev. B 102, 020408 (2020).

[28] A. Ghosh, M. Palit, S. Maity, V. Dwij, S. Rana, and S. Datta, Spin-phonon coupling and magnon scattering in few-layer antiferromagnetic $\mathrm{FePS}_{3}$, Phys. Rev. B 103, 064431 (2021).

[29] A. Hoffmann and S. D. Bader, Opportunities at the Frontiers of Spintronics, Phys. Rev. Appl. 4, 047001 (2015).

[30] J. Li, T. Higuchi, N. Kanda, K. Konishi, S. G. Tikhodeev, and M. Kuwata-Gonokami, Control of magnetic dipole terahertz radiation by cavity-based phase modulation, Opt. Express 19, 22550 (2011).

[31] M. Białek, A. Magrez, and J.-P. Ansermet, Spin-wave coupling to electromagnetic cavity fields in dysposium ferrite, Phys. Rev. B 101, 024405 (2020).

[32] J. R. Everts, G. G. G. King, N. J. Lambert, S. Kocsis, S. Rogge, and J. J. Longdell, Ultrastrong coupling between a microwave resonator and antiferromagnetic resonances of rare-earth ion spins, Phys. Rev. B 101, 214414 (2020).

[33] K. Grishunin, T. Huisman, G. Q. Li, E. Mishina, T. Rasing, A. V. Kimel, K. Zhang, Z. M. Jin, S. X. Cao, W. Ren, G. H. Ma, and R. V. Mikhaylovskiy, Terahertz magnon-polaritons in $\mathrm{TmFeO}_{3}$, ACS Photonics 5, 1375 (2018).

[34] L. Y. Shi, D. Wu, Z. X. Wang, T. Lin, C. M. Hu, and N. L. Wang, Revealing ultra-strong magnon-photon coupling in a polar antiferromagnet $\mathrm{Fe}_{2} \mathrm{Mo}_{3} \mathrm{O}_{8}$ by time domain terahertz spectroscopy, arXiv:2004.05823 [cond-mat.str-el] (2020).

[35] X. Li, M. Bamba, N. Yuan, Q. Zhang, Y. Zhao, M. Xiang, K. Xu, Z. Jin, W. Ren, G. Ma, S. Cao, D. Turchinovich, and J. Kono, Observation of Dicke cooperativity in magnetic interactions, Science 361, 794 (2018).

[36] P. Sivarajah, A. Steinbacher, B. Dastrup, J. Lu, M. Xiang, W. Ren, S. Kamba, S. Cao, and K. A. Nelson, THz-frequency magnon-phonon-polaritons in the collective strong-coupling regime, J. Appl. Phys. 125, 213103 (2019).

[37] C.-M. Hu, Chapter four - the 2020 roadmap for spin cavitronics, Solid State Phys. 71, 117 (2020).

[38] R. Przeniosło, I. Sosnowska, M. Stekiel, D. Wardecki, A. Fitch, and J. B. Jasiński, Monoclinic deformation of the crystal lattice of hematite $\alpha-\mathrm{Fe}_{2} \mathrm{O}_{3}$, Phys. B: Condens. Matter 449, 72 (2014).

[39] F. J. Morin, Magnetic susceptibility of $\alpha \mathrm{Fe}_{2} \mathrm{O}_{3}$ and $\alpha \mathrm{Fe}_{2} \mathrm{O}_{3}$ with added titanium, Phys. Rev. 78, 819 (1950). 
[40] K. S. Aleksandrov, L. N. Bezmaternykh, G. V. Kozlov, S. P. Lebedev, A. A. Mukhin, and A. S. Prokhorov, Anomalies of high-frequency magnetic permeability of hematite at the Morin phase transition, J. Exp. Theor. Phys. 65, 591 (1986).

[41] I. Dzyaloshinsky, A thermodynamic theory of "weak" ferromagnetism of antiferromagnetics, J. Phys. Chem. Solids 4, 241 (1958).

[42] C. Caspers, V. P. Gandhi, A. Magrez, E. de Rijk, and J.-P. Ansermet, Sub-terahertz spectroscopy of magnetic resonance in $\mathrm{BiFeO}_{3}$ using a vector network analyzer, Appl. Phys. Lett. 108, 241109 (2016).

[43] M. Białek, A. Magrez, A. Murk, and J.-P. Ansermet, Spinwave resonances in bismuth orthoferrite at high temperatures, Phys. Rev. B 97, 054410 (2018).

[44] M. Białek, T. Ito, H. Rønnow, and J.-P. Ansermet, Terahertz-optical properties of a bismuth ferrite single crystal, Phys. Rev. B 99, 064429 (2019).

[45] J. Zhang, M. Białek, A. Magrez, H. Yu, and J.-P. Ansermet, Antiferromagnetic resonance in $\mathrm{TmFeO}_{3}$ at high temperatures, J. Magn. Magn. Mater. 523, 167562 (2021).

[46] M. Harder, L.-H. Bai, C. Match, J. Sirker, and C.-M. $\mathrm{Hu}$, Study of the cavity-magnon-polariton transmission line shape, Sci. China Phys. Mech. Astron. 59, 117511 (2016).

[47] D. L. Mills and E. Burstein, Polaritons: The electromagnetic modes of media, Rep. Prog. Phys. 37, 817 (1974).
[48] O. O. Soykal and M. E. Flatté, Strong Field Interactions between a Nanomagnet and a Photonic Cavity, Phys. Rev. Lett. 104, 077202 (2010).

[49] T. Niemczyk, F. Deppe, M. Mariantoni, E. P. Menzel, E. Hoffmann, G. Wild, L. Eggenstein, A. Marx, and R. Gross, Fabrication technology of and symmetry breaking in superconducting quantum circuits, Supercond. Sci. Technol. 22, 034009 (2009).

[50] G. Flower, M. Goryachev, J. Bourhill, and M. E. Tobar, Experimental implementations of cavity-magnon systems: From ultra strong coupling to applications in precision measurement, New J. Phys. 21, 095004 (2019).

[51] J. Bourhill, V. Castel, A. Manchec, and G. Cochet, Universal characterization of cavity-magnon polariton coupling strength verified in modifiable microwave cavity, J. Appl. Phys. 128, 073904 (2020).

[52] N. Pailhé, J. Majimel, S. Pechev, P. Gravereau, M. Gaudon, and A. Demourgues, Investigation of nanocrystallized $\alpha$ $\mathrm{Fe}_{2} \mathrm{O}_{3}$ prepared by a precipitation process, J. Phys. Chem. C 112, 19217 (2008).

[53] C. G. Shull, W. A. Strauser, and E. O. Wollan, Neutron diffraction by paramagnetic and antiferromagnetic substances, Phys. Rev. 83, 333 (1951).

[54] M. Białek, J. Zhang, H. Yu, and J.-P. Ansermet (to be published 2021).

[55] D. M. Pozar, Microwave Engineering (Wiley, Hoboken, 2011). 